\title{
The Association between dietary total antioxidant capacity with the risk of helicobacter pylori infection among adult: a case-control study
}

Mohammadhassan Sohouli ( $\sim$ mohammadhassansohouli@gmail.com )

Iran University of Medical Sciences

Niloufar Haghshenas

Iran University of Medical Sciences

Somaye Fatahi

Iran University of Medical Sciences

\section{Research}

Keywords: Dietary total antioxidant capacity, Obesity, Chronic Diseases, Helicobacter pylori

Posted Date: June 16th, 2020

DOI: https://doi.org/10.21203/rs.3.rs-26732/v1

License: (c) (i) This work is licensed under a Creative Commons Attribution 4.0 International License.

Read Full License 


\section{Abstract \\ Objective}

Dietary total antioxidant capacity (TAC) has been assumed as a useful tool to assess the relationship between the cumulative antioxidant food capacity and several chronic disorders. The aim of this study was to evaluate the association between DTAC and risk of Helicobacter pylori in a case-control study.

\section{Methods}

This case-control study was carried out among 150 patients with Helicobacter pylori and 302 healthy subjects aged 18-55 years. Dietary data were collected using a validated 168- items quantitative food frequency questionnaire. DTAC was calculated based on the ferric reducing-antioxidant power (FRAP) values that reported by US Department of Agriculture.

\section{Results}

The mean age and BMI of the study participants were 38.70 years and 25.86 , respectively. Compared with control subjects, participants with $\mathrm{H}$ pylori significantly were older and had higher BMI and smoking use. Patients with $\mathrm{H}$ pylori compared with control individuals had also lower intake of total fiber, vitamin $\mathrm{E}$, vitamin $C$, vitamin $D$, total dairy, whole grain and vegetables. Compared with participants in the lowest tertile of DTAC, those in the highest terrtile had a significantly lower OR for $\mathrm{H}$ pylori after further adjustment for potential confounders (Model 2: OR, 0.20; 95\% Cl, 0.10-0.40; P for trend, < 0.001 ).

\section{Conclusions}

Our findings showed that a high DTAC was associated with a reduced risk of Helicobacter pylori in adult, which reflects the increased intake of natural dietary antioxidants to prevent the progression of Helicobacter pylori. However, further studies are needed to investigate the trelation between DTAC and risk of Helicobacter pylori.

\section{Introduction}

Helicobacter pylori (H pylori), which it has been estimated that its worldwide prevalence is approximately 4.4 billion individuals(1), is a gram-negative micro-aerophilic pathogen replicating on the surface of the gastric epithelium that can cause gastritis, gastric cancer(2). Moreover, the recent document showed that the $\mathrm{H}$ pylori infection is associated with an increased risk of pancreatic disease(3), lymphoma (4) and atherosclerosis(5). Based on evidence, $\mathrm{H}$ pylori infection is affected by genetic and environmental factors (6) such as host diet and nutrition status(7). 
On other hand, Studies suggest that antioxidants (eg, vitamins and N-acetylcysteine) play a role in reducing active oxygen species and gastric inflammation caused by $\mathrm{H}$. pylori, which also exacerbates the disease and prevents the growth of bacteria and eliminates biofilm formation(8). So far, numerous studies have shown that vitamin $\mathrm{C}$ and $\mathrm{E}$ levels are lower in people with Helicobacter pylori than in those without H. pylori(8). Thus, impaired antioxidant and anti-inflammatory defenses may cause or exacerbate a chronic condition of pyloric $\mathrm{H}$ infection. On other hand, various aspects of the effect of natural antioxidants found different in foods on the modulation of oxidative stress and disorders in the antioxidant system have been studied. $(9,10)$ However, the evaluation of an antioxidant compound alone cannot reflect the total antioxidant potency of the diet and reflect the synergistic and potential effects of dietary antioxidant interactions. Thus, the term total antioxidant capacity for diet (DTAC)has been developed and is used as a suitable tool to evaluate the effects of dietary antioxidants that is a strong correlation with serum total antioxidant capacity (11) And it is closely related to the quality of the diet to determine the risk of chronic diseases (12).

Several evidences have suggested the potential link between DTAC and reduce the risk of chronic diseases such as diabetes(13), metabolic and oxidative stress markers(14), ulcerative colitis(15), blood pressure(16), and cardiovascular disease(17), which share common metabolic parameters with $\mathrm{H}$ pylori. However, to our knowledge, the association between DTAC and risk of development $\mathrm{H}$ pylori have not yet been investigated. Only, a number of studies in vivo and animal studies have shown the ability of nutrients rich in antioxidant to reduced oxidative stress and subsequently reduced risk of $\mathrm{H}$ pylori including garlic, ginger, quercetin, green tea and Cranberry (18-21). However, the synergistic effects of different antioxidant nutrients, which also found in DTAC and other chemical ingredients utilized in the growing or preparation of food have not been discussed.(22).

Considering the lack of convincing evidence regarding the association of DTAC with liver function, in this study we aimed to investigate the association between DTAC and risk of $\mathrm{H}$ pylori in Iranian population in order to improve and reduce the burden of diseases.

\section{Subjects and Methods}

\section{Subjects And Methods}

\section{Participants}

This case-control study was performed on 153 patients with $H$ pylori and 306 healthy subjects who had been referred to the Hazrat Rasoul Hospital, Tehran, Iran during the recent years. In this study, patients with $\mathrm{H}$ pylori infection were confirmed by one of the diagnostic methods of blood antibody, fecal antigen, UBT respiratory test, endoscopy and gastric biopsy and performed Rapid Urease Test or pathological and microscopic examination. If they meet the inclusion criteria, are selected as case group after obtaining written informed consent. The control group was twice as likely as the case group and among other patients referred to other parts of the hospital such as ophthalmology, orthopedics, maxillofacial surgery, 
ear, nose, and throat who were not diagnosed $H$ pylori infection with the diagnostic methods and also not having a history of alcohol or alcohol consumption less than $10 \mathrm{mg} /$ day in women and less than $20 \mathrm{mg}$ / day in men. Case and control were matched regarding age and gender. After entering into the study, information about demographic variables and Hematological parameters were obtained by completing the General Information Questionnaire. Also, the Hematological parameters checked after they came to the hospital, again. Then, for completing data on dietary intake and other information we called the patients to invite them to the research center in a special date. Participants with a history of certain diseases (Gastrointestinal malignant diseases, psychiatric psychosis, diseases that result in lack of reminder like Alzheimer's) were excluded. Also, pregnant and lactating women and subjects with an arbitrary special diet were excluded. In this study, nutritionists were used as interviewers in order to reduce information bias as well as to answer completely to the survey questions. Also, in order to assess physical activity levels of participants, we used the General Practice Physical Activity Questionnaire (GPPAQ), a simple questionnaire reflecting an individual's current physical activity (23).

\section{Anthropometric assessment}

Anthropometric measurements were conducted by a trained dietician. Weight was measured using a standard digital seca scale (made in Germany), while participants wore minimum clothes and without shoes and recorded to the nearest $100 \mathrm{~g}$. Height was measured using a mounted tape in a standing relaxed shoulder position with no shoes to the nearest $0.5 \mathrm{~cm}$. Body mass index (BMI) was calculated as weight $(\mathrm{kg})$ divided by height in square meters $(\mathrm{m} 2)$.

\section{Measurement of Hematological parameters}

All stool samples were collected in plastic containers and sent to the laboratory within $2 \mathrm{~h}$. About $5 \mathrm{~mL}$ of venous blood were collected from each subject (cases and controls) and divided equally $(2.5 \mathrm{~mL})$ in one tube containing K3-ethylenediaminetetraacetic acid (K3-EDTA) to perform a complete blood count (CBC) using a Cell Dyne 1800 electronic counter (Sequoia-Turner Corporation, California, USA) and in a serum tube to determine serum iron using the DiaSys reagent kit. The serum vitamin B12 concentration was determined quantitatively using a solid phase, competitive chemiluminescent enzyme immunoassay (Immulite/Immulite 1000).

\section{Dietary assessment and DTAC calculation}

Nutritional status of individuals was assessed by a 168- items FFQ. The questionnaire has a standard size for each food item designed according to the Willett method (24). Study participants were asked to indicate their frequency of consumption of each food item according to their consumption in the past year. Depending on the type of food consumed, the frequency of consumption per day, week or month was questioned. Previous studies using this questionnaire have provided acceptable results and validity of the questionnaire indicates that the questionnaire has acceptable validity. The values listed for each food item were converted to grams daily using the Home Scale Handbook. Then, the amount of nutrients obtained by each individual was obtained using Nutritionist IV software. The total antioxidant capacity of 
the diet was obtained from previous articles based on the ferric reducing-antioxidant power (FRAP). The FRAP is defined as the ability of dietary antioxidants to reduce ferric to ferrous ions. The FRAP was expressed in milligrams per 100 grams of food(25). For similar foods in Iranian culture (for example, different types of bread), the average total values will be calculated. Finally, the frequency of consumption of each food item is multiplied by the amount of iron-reducing antioxidant power associated with it, and then the diet is collected for each participant.to obtain the total antioxidant capacity

\section{Statistical analysis.}

All statistical analyses were using SPSS software (version 19.0; SPSS Inc, Chicago IL). The normality of variables was evaluated by Kolmogorov-Smirnov and histogram tests. For the variables that do not have a normal distribution, logarithmic equivalent (Ln transformation) of that variable was used in the analysis. Also, independent sample T test was used for comparing the mean of DTAC as well as the general characteristics and hematological parameters among case and control group. For assessing the relation between DTAC index and $H$ pylori in an adjusted model, multiple logistic regressions were used. The data were presented as mean \pm standard deviation and odds ratio with $95 \%$ confidence interval, and in all results, the significance level was determined as $\mathrm{P}<0.05$.

\section{Result}

The mean \pm SD for the age and BMI of the study population (59.5\% women) were $38.70 \pm 10.65$ years and $25.86 \pm 4.84 \mathrm{Kg} . \mathrm{m} 2$, respectively.

Table 1 shows the distribution of cases and controls according to socio-demographic characteristics, smoking habits, alcohol drinking, body mass composition and hematological parameters. Compared with control subjects, participants with H pylori significantly were older and had higher BMI and smoking use. The mean levels of hemoglobin, RBC, WBC, hematocrit, and mean corpuscular hemoglobin concentration (MCHC) were significantly lower among cases compared to controls individuals. Furthermore, no significant differences were found for mean corpuscular volume (MCV) and mean corpuscular hemoglobin $(\mathrm{MCH})$ cases and controls subjects. On comparing cases with controls, the mean levels of serum vitamin B12 and iron were significantly lower in the patients.

Table 2 illustrates the dietary intake of $H$ pylori patients compared to controls. Patients with $H$ pylori compared with control individuals had also higher intake of energy, protein, carbohydrate, saturated fatty acids, fructose, iron, whole and refine grain, and red and processed meat and lower intake of total fiber, vitamin $E$, vitamin $C$, vitamin $D$, total dairy, Whole grain and vegetables. There were no significant differences in all other characteristics and dietary intake among case and control subjects.

The ORs and $95 \%$ Cls for $\mathrm{H}$ pylori in the terrtiles of DTAC are shown in Table 3. Compared with participants in the lowest tertile of DTAC, those in the highest terrtile had a significantly lower OR for $\mathrm{H}$ pylori (crude model: OR, 0.29; $95 \% \mathrm{Cl}, 0.14-0.6$; P for trend, 0.001), which remained significant after 
further adjustment for BMI, waist circumference, physical activity, smoking, dietary intake of energy and fat. (Model 2: OR, $0.20 ; 95 \% \mathrm{Cl}, 0.10-0.40 ; \mathrm{P}$ for trend, $<0.001$ ).

\section{Discussion}

The study investigated the association between DTAC and $\mathrm{H}$ pylori in a case-control study. We found that DTAC had an inverse association with risk of $\mathrm{H}$ pylori infection. Participants with higher DTAC score had a significantly lower OR for H pylori infection compared with low DTAC score, after adjustment for potential factors. Our study suggests that DTAC may potentially reduce the risk of NAFLD.

According to our information, this is the first time that the inverse relationship between DTAC and risk of $\mathrm{H}$ pylori infection has been proven $\mathrm{H}$ pylori infection has been documented. In the previous evidences, dietary TAC has been inversely associated with several chronic diseases such as cardiovascular diseases(17), cancer (26)diabetes (13), and metabolic disorders(14), which share common metabolic parameters with $\mathrm{H}$ pylori infection.

Overall, an inverse associations between intake of antioxidant micronutrients and antioxidant-rich foods with risk of $\mathrm{H}$ pylori infection was registered. For example, The DASH diet (Dietary Approaches to Stop Hypertension), dietary patterns with antioxidant-rich component based on fruit and vegetables, low-fat dairy products, and whole grains, has been shown to eradicate Helicobacter pylori and its gastric cancer(27), as well as an inverse association in chronic diseases associated with $\mathrm{H}$ pylori infection (28). Furthermore, coffee has been investigated as an antioxidant-rich nutrient as a risk reduction for coffee. So that, Coffee showed a strong antibacterial action against Helicobacter pylori (H. pylori)(29).This action against $\mathrm{H}$. pylon suggests that coffee may have action as a useful natural inhibitor of gastritis and gastric ulcers. Also, the ability of other antioxidant-rich nutrient to increase plasma TAC and finally reduced risk of $\mathrm{H}$ pylori infection through several mechanism, garlic, ginger, quercetin, green tea and Cranberry (19-22) was well revealed. The findings were confirmed even in complementary interventions with antioxidants. Besides, the beneficial effects of the combination of antioxidant compounds were also reported, as demonstrated the synergistic effect of Simultaneous administration and combination of one or more antioxidant supplements reducing risk of $\mathrm{H}$ pylori infection(19).

The mechanism of action of dietary antioxidant compounds on $\mathrm{H}$. pylori is not clear. However, the intake of antioxidant-rich nutrients in animals with Helicobacter pylori has been shown to reduce both the number of bacteria in the gastric mucosa and the inflammatory process $(8,20)$. On the other hand, it is possible that these cultivations, by destroying the Iklova factor, have direct antiseptic properties and prevent NF-к $\beta$ nuclear transfer, thereby reducing genetic expression and producing mediators associated with relevant inflammation such as TNF-a, IL-1 $\beta$, IL- 6 and IL-8. It is possible that antioxidant-rich nutrient, by controlling some of the toxins that cause enough bacteria to grow by creating special anionic channels in the plasma membrane associated with the release of bicarbonate and organic anions in gastric cells, they have a beneficial effect(30). On the other hand, it is possible that these compounds reduce the action of urease on $\mathrm{H}$. pylori, because it has been shown that several polyphenolic compounds 
can alter the activity of this enzyme, which is responsible for the survival of bacteria in acid.(31) In addition, fiber, as part of a healthy diet in many high-antioxidant plant foods, may have health benefits through protection against oxidative damage, as well as improving glucose metabolism and serum lipids (32). Also, some prior studies have declared that dietary fibers can alter abundances of specific gut microbes and change the profile of human gut microbiota (33-36). Fiber fermentation is dependent on microbiota (37) and it has been seen that fiber may increase gram-positive bacteria (38), by decreasing the gut transit time and $\mathrm{pH}(39,40)$. Moreover, we know that $H$ pylori is the gram-negative bacteria so it may be negatively affected by fiber. Also, a high fiber diet in rich of vegetables, fruits, whole grains and beans $(41)$ can exert a protective effect against $H$ pylori infection $(42,43)$. This may be due to their prebiotic properties which is confirmed in a meta-analysis (44). Besides, fresh vegetables are the source of vitamin $\mathrm{C}$ which has been mentioned as a chemopreventive factor against digestive disorders caused by $\mathrm{H}$ pylori infection (42). As far as we know, vitamin $\mathrm{C}$ can lessen stomach cancer risk and exert an effect on the cycle of $H$ pylori infection (45). Vitamin $\mathrm{C}$ is highly concentrated in stomach mucosa and gastric juice. it has a positive effect on the production and function of immune cells and immunoglobulin (46). In our study, dietary vitamin c consumption was significantly lesser in case group. Also, Dietary antioxidants may reduce the risk of developing this disease by reducing the body's obesity and visceral fat, which are known to be one of the risk factors for Helicobacter pylori infection $(47,48)$.

This is the first study to assess the relationships between DTAC with risk of $\mathrm{H}$ pylori Our sample size was sufficient and we tried to eliminate the effect of confounders as far as possible, by adjusting wide range of variables and a validated questionnaire has been used. As for most case-control studies, the causal relationship can be determined but it cannot be quite definite. Furthermore, some conditions of foods such as the growing condition, cultivation method, storage, processing and cooking condition, and assay method might affect the antioxidants content of the foods (25) Also, information collecting in these kinds of studies are based on individual's reports and memory which is not totally reliable. We have tried to minimize information bias by administrating a questionnaire by trained interviewers.

\section{Conclusion}

Our findings showed that a high DTAC was associated with a reduced risk of $H$ pylori infection in adult, which reflects the increased intake of natural dietary antioxidants to prevent the progression of. However, further observational studies and clinical trials are needed to investigate the exact relation between DTAC and risk of $H$ pylori infection.

\section{Declarations}

\section{Acknowledgements}

We express our appreciation to the participants of this study.

\section{Disclosure statement}


None of the authors had any personal or financial conflicts of interest.

\section{Authors' contributions}

S.F, and Mh.S contributed in conception, design, and statistical analysis. S.N, and N.H contributed in data collection and manuscript drafting. N. H and Mh.S supervised the study. All authors approved the final version of the manuscript.

\section{Funding}

No funding

\section{Availability of data and materials}

The datasets used and/or analyzed during the current study are available from the corresponding author on reasonable request.

\section{Ethics approval and consent to participate}

Not applicable.

\section{Consent for publication}

All authors of this manuscript declared their consent for publication.

\section{Competing interests}

The authors declare that they have no competing interests.

\section{References}

1. Hooi JK, Lai WY, Ng WK, Suen MM, Underwood FE, Tanyingoh D, et al. Global prevalence of Helicobacter pylori infection: systematic review and meta-analysis. Gastroenterology. 2017;153(2):420-9.

2. Brown LM. Helicobacter pylori: epidemiology and routes of transmission. Epidemiol Rev. 2000;22(2):283-97.

3. Bulajic M, Panic N, Löhr JM. Helicobacter pylori and pancreatic diseases. World journal of gastrointestinal pathophysiology. 2014;5(4):380.

4. Parsonnet J, Hansen S, Rodriguez L, Gelb AB, Warnke RA, Jellum E, et al. Helicobacter pylori infection and gastric lymphoma. N Engl J Med. 1994;330(18):1267-71.

5. Vijayvergiya R, Vadivelu R. Role of Helicobacter pylori infection in pathogenesis of atherosclerosis. World J Cardiol. 2015;7(3):134. 
6. Malaty HM, Engstrand L, Pedersen NL, Graham DY. Helicobacter pylori infection: genetic and environmental influences: a study of twins. Ann Intern Med. 1994;120(12):982-6.

7. Haley KP, Gaddy JA. Nutrition and Helicobacter pylori: host diet and nutritional immunity influence bacterial virulence and disease outcome. Gastroenterology research and practice. 2016;2016.

8. Yang-Ou YB, Hu Y, Zhu Y, Lu NH. The effect of antioxidants on Helicobacter pylori eradication: A systematic review with meta-analysis. Helicobacter. 2018;23(6):e12535.

9. Serafini M, Del Rio D. Understanding the association between dietary antioxidants, redox status and disease: is the total antioxidant capacity the right tool? Redox report. 2004;9(3):145-52.

10. Meydani M. Dietary antioxidants modulation of aging and immune-endothelial cell interaction. Mech Ageing Dev. 1999;111(2-3):123-32.

11. Cao G, Prior RL. Comparison of different analytical methods for assessing total antioxidant capacity of human serum. Clinical chemistry. 1998;44(6):1309-15.

12. Wu X, Gu L, Holden J, Haytowitz DB, Gebhardt SE, Beecher G, et al. Development of a database for total antioxidant capacity in foods: a preliminary study. Journal of Food composition analysis. 2004;17(3-4):407-22.

13. Mancini FR, Affret A, Dow C, Balkau B, Bonnet F, Boutron-Ruault M-C, et al. Dietary antioxidant capacity and risk of type 2 diabetes in the large prospective E3N-EPIC cohort. Diabetologia. 2018;61(2):308-16.

14. Hermsdorff HHM, Puchau B, Volp ACP, Barbosa KB, Bressan J, Zulet M, et al. Dietary total antioxidant capacity is inversely related to central adiposity as well as to metabolic and oxidative stress markers in healthy young adults. Nutrition metabolism. 2011;8(1):59.

15. Koch TR, Yuan L-X, Stryker SJ, Ratliff P, Telford GL, Opara EC. Total antioxidant capacity of colon in patients with chronic ulcerative colitis. Digestive diseases sciences. 2000;45(9):1814-9.

16. Villaverde P, Lajous M, MacDonald C-J, Fagherazzi G, Bonnet F, Boutron-Ruault M-C. High dietary total antioxidant capacity is associated with a reduced risk of hypertension in French women. Nutrition journal. 2019;18(1):31.

17. Rautiainen S, Levitan EB, Orsini N, Åkesson A, Morgenstern R, Mittleman MA, et al. Total antioxidant capacity from diet and risk of myocardial infarction: a prospective cohort of women. Am J Med. 2012;125(10):974-80.

18. Gaus K, Huang Y, Israel DA, Pendland SL, Adeniyi BA, Mahady GB. Standardized ginger (Zingiber officinale) extract reduces bacterial load and suppresses acute and chronic inflammation in Mongolian gerbils infected with cagA + Helicobacter pylori. Pharmaceutical biology. 2009;47(1):928.

19. Vattem D, Lin Y-T, Ghaedian R, Shetty K. Cranberry synergies for dietary management of Helicobacter pylori infections. Process Biochem. 2005;40(5):1583-92.

20. González-Segovia R, Quintanar JL, Salinas E, Ceballos-Salazar R, Aviles-Jiménez F, Torres-López J. Effect of the flavonoid quercetin on inflammation and lipid peroxidation induced by Helicobacter pylori in gastric mucosa of guinea pig. Journal of gastroenterology. 2008;43(6):441. 
21. Jeong M, Park JM, Han YM, Kangwan N, Kwon SO, Kim BN, et al. Dietary Intervention of Artemisia and Green Tea Extracts to Rejuvenate Helicobacter pylori-Associated Chronic Atrophic Gastritis and to Prevent Tumorigenesis. Helicobacter. 2016;21(1):40-59.

22. de Oliveira DG, de Faria Ghetti F, Moreira APB, Hermsdorff HHM, de Oliveira JM, de Castro LEVV. Association between dietary total antioxidant capacity and hepatocellular ballooning in nonalcoholic steatohepatitis: a cross-sectional study. Eur J Nutr. 2019;58(6):2263-70.

23. Ahmad S, Harris T, Limb E, Kerry S, Victor C, Ekelund U, et al. Evaluation of reliability and validity of the General Practice Physical Activity Questionnaire (GPPAQ) in 60-74 year old primary care patients. BMC Fam Pract. 2015;16(1):113.

24. Azadbakht L, Esmaillzadeh A. Red meat intake is associated with metabolic syndrome and the plasma C-reactive protein concentration in women. J Nutr. 2009;139(2):335-9.

25. Haytowitz DB, Bhagwat S. USDA database for the oxygen radical absorbance capacity (ORAC) of selected foods, Release 2. US Department of Agriculture. 2010:10-48.

26. Vece MM, Agnoli C, Grioni S, Sieri S, Pala V, Pellegrini N, et al. Dietary total antioxidant capacity and colorectal cancer in the Italian EPIC cohort. PLoS One. 2015;10(11).

27. Onvani S, Haghighatdoost F, Azadbakht L. Dietary approach to stop hypertension (DASH): diet components may be related to lower prevalence of different kinds of cancer: A review on the related documents. Journal of research in medical sciences: the official journal of Isfahan University of Medical Sciences. 2015;20(7):707.

28. Liese AD, Nichols M, Sun X, D'Agostino RB, Haffner SM. Adherence to the DASH Diet is inversely associated with incidence of type 2 diabetes: the insulin resistance atherosclerosis study. Diabetes Care. 2009;32(8):1434-6.

29. Okabe Y, Yamamoto Y, Yasuda K, Hochito K, Kawano K, Ishii N. The antibacterial effects of coffee on Escherichia coli and Helicobacter pylori. Journal of Clinical Biochemistry Nutrition. 2004;34(3):85-7.

30. Tombola F, Campello S, De Luca L, Ruggiero P, Del Giudice G, Papini E, et al. Plant polyphenols inhibit VacA, a toxin secreted by the gastric pathogen Helicobacter pylori. FEBS Lett. 2003;543(1-3):184-9.

31. Xiao Z-P, Shi D-H, Li H-Q, Zhang L-N, Xu C, Zhu H-L. Polyphenols based on isoflavones as inhibitors of Helicobacter pylori urease. Bioorg Med Chem. 2007;15(11):3703-10.

32. Valko M, Leibfritz D, Moncol J, Cronin MT, Mazur M, Telser J. Free radicals and antioxidants in normal physiological functions and human disease. Int J Biochem Cell Biol. 2007;39(1):44-84.

33. Tuohy KM, Conterno L, Gasperotti M, Viola R. Up-regulating the human intestinal microbiome using whole plant foods, polyphenols, and/or fiber. J Agric Food Chem. 2012;60(36):8776-82.

34. De Filippo C, Cavalieri D, Di Paola M, Ramazzotti M, Poullet JB, Massart S, et al. Impact of diet in shaping gut microbiota revealed by a comparative study in children from Europe and rural Africa. Proceedings of the National Academy of Sciences. 2010;107(33):14691-6.

35. Dominianni C, Sinha R, Goedert JJ, Pei Z, Yang L, Hayes RB, et al. Sex, body mass index, and dietary fiber intake influence the human gut microbiome. PloS one. 2015;10(4). 
36. Holscher HD, Caporaso JG, Hooda S, Brulc JM, Fahey GC Jr, Swanson KS. Fiber supplementation influences phylogenetic structure and functional capacity of the human intestinal microbiome: follow-up of a randomized controlled trial. Am J Clin Nutr. 2015;101(1):55-64.

37. Yin R, Kuo H-C, Hudlikar R, Sargsyan D, Li S, Wang L, et al. Gut Microbiota, Dietary Phytochemicals, and Benefits to Human Health. Current Pharmacology Reports. 2019;5(5):332-44.

38. Duncan SH, Louis $\mathrm{P}$, Thomson JM, Flint HJ. The role of $\mathrm{pH}$ in determining the species composition of the human colonic microbiota. Environ Microbiol. 2009;11(8):2112-22.

39. Probert C, Emmett P, Heaton K. Some determinants of whole-gut transit time: a population-based study. QJM: An International Journal of Medicine. 1995;88(5):311-5.

40. Lewis S, Heaton K. Increasing butyrate concentration in the distal colon by accelerating intestinal transit. Gut. 1997;41(2):245-51.

41. Mcintosh M, Miller C. A Diet Containing Food Rich in Soluble and Insoluble Fiber Improves Glycemic Control and Reduces Hyperlipidemia among Patients with Type 2 Diabetes Mellitus. Nutr Rev. 2001;59(2):52-5.

42. Izzotti A, Durando P, Ansaldi F, Gianiorio F, Pulliero A. Interaction between Helicobacter pylori, diet, and genetic polymorphisms as related to non-cancer diseases. Mutation Research/Fundamental Molecular Mechanisms of Mutagenesis. 2009;667(1-2):142-57.

43. Wang $T$, Cai $H$, Sasazuki S, Tsugane $S$, Zheng W, Cho ER, et al. Fruit and vegetable consumption, Helicobacter pylori antibodies, and gastric cancer risk: A pooled analysis of prospective studies in China, Japan, and Korea. International journal of cancer. 2017;140(3):591-9.

44. Khan MSA, Khundmiri SUK, Khundmiri SR, Al-Sanea MM, Mok PL. Fruit-derived polysaccharides and terpenoids: Recent update on the gastroprotective effects and mechanisms. Front Pharmacol. 2018;9:569.

45. Shi L-Q, Zheng R-L. DNA damage and oxidative stress induced by Helicobacter pylori in gastric epithelial cells: protection by vitamin $\mathrm{C}$ and sodium selenite. Die Pharmazie-An International Journal of Pharmaceutical Sciences. 2006;61(7):631-7.

46. Ruiz B, Rood JC, Fontham ET, Malcom GT, Hunter FM, Sobhan M, et al. Vitamin C concentration in gastric juice before and after anti-Helicobacter pylori treatment. American Journal of Gastroenterology. 1994;89(4).

47. Xu M-Y, Liu L, Yuan B-S, Yin J, Lu Q-B. Association of obesity with Helicobacter pylori infection: A retrospective study. World journal of gastroenterology. 2017;23(15):2750.

48. Bahadoran Z, Golzarand M, Mirmiran P, Shiva N, Azizi F. Dietary total antioxidant capacity and the occurrence of metabolic syndrome and its components after a 3-year follow-up in adults: Tehran Lipid and Glucose Study. Nutrition metabolism. 2012;9(1):70.

\section{Tables}

Table 1: Baseline characteristics and Hematological parameters of the study participants 


\begin{tabular}{|c|c|c|c|}
\hline & case $(n=150)$ & control $(n=302)$ & P-value \\
\hline \multicolumn{4}{|l|}{ Demographic variables } \\
\hline Age (year) & $42.0(13.5)$ & $37.0(8.4)$ & $<0.001$ \\
\hline Male, N (\%) & $48(32.0)$ & $135(44.7)$ & 0.01 \\
\hline BMI $\left(\mathrm{Kg} / \mathrm{m}^{2}\right)$ & $28.0(6.5)$ & $24.7(3.2)$ & $<0.001$ \\
\hline \multicolumn{4}{|l|}{ Physical activity, N (\%) } \\
\hline Low & $114(76.0)$ & $220(72.8)$ & 0.484 \\
\hline Moderate & $29(19.3)$ & $59(19.5)$ & 0.465 \\
\hline High & $7(4.7)$ & $23(7.6)$ & 0.362 \\
\hline Current smokers, N (\%) & $11(7.3)$ & $8(2.6)$ & 0.019 \\
\hline Alcohol drinking, N (\%) & $16(10.7)$ & $21(7.0)$ & 0.175 \\
\hline $\mathrm{Hb}(\mathrm{g} / \mathrm{dL})$ & $11.2(3.2)$ & $13.6(2.9)$ & 0.001 \\
\hline $\mathrm{RBC}(\times 109 / \mathrm{L})$ & $4.1(1.2)$ & $4.6(1.2)$ & $<0.001$ \\
\hline WBC $(\times 109 / L)$ & $6.8(2.3)$ & $7.3(2.5)$ & 0.049 \\
\hline Hct (\%) & $37.8(6.9)$ & $43.0(6.7)$ & $<0.001$ \\
\hline $\operatorname{MCV}(f L)$ & $85.2(12.3)$ & $84.8(7.9)$ & 0.589 \\
\hline $\mathrm{MCH}(\mathrm{pg})$ & $30.1(3.5)$ & $30.0(2.6)$ & 0.651 \\
\hline $\mathrm{MCHC}(\mathrm{g} / \mathrm{dL})$ & $33.4(2.5)$ & $34.0(1.3)$ & 0.005 \\
\hline Vitamin B12 $(\mathrm{pg} / \mathrm{mL})$ & $223.4(98.7)$ & $365.3(130.6)$ & 0.001 \\
\hline Iron $(\mathrm{g} / \mathrm{dL})$ & $63.9(19.8)$ & $74.2(16.3)$ & 0.001 \\
\hline
\end{tabular}

WBC: white blood cell count; RBC: red blood cell count; Hb: hemoglobin; Hct: hematocrit; MCV: mean corpuscular volume; $\mathrm{MCH}$ : mean corpuscular hemoglobin; $\mathrm{MCHC}$ : mean corpuscular hemoglobin concentration.

$p<0.05$ : significant.

Values are expressed as means \pm standard deviation (SD) of 150 subjects.

$P$ values are resulted from chi square and student t-test.

Table 2. Dietary intake among the Case and Control Groups 


\begin{tabular}{|llll|}
\hline & case (n=150) & control (n=302) & P-value \\
\hline Dietary Intake & & & \\
\hline Energy(Kcal/d) & $2629.1(779.9)$ & $2282.9(638.6)$ & $<0.001$ \\
\hline Carbohydrate(gr/d) & $393.2(122.3)$ & $325.1(103.2)$ & $<0.001$ \\
\hline Protein(gr/d) & $94.5(32.2)$ & $76.4(24.2)$ & $<0.001$ \\
\hline Fat(g/d) & $83.8(30.2)$ & $81.3(29.2)$ & 0.398 \\
\hline Saturated fatty acids (gr/d) & $27.5(10.6)$ & $24.3(9.2)$ & 0.001 \\
\hline Mono unsaturated fatty acids & $27.2(9.5)$ & $28.0(10.6)$ & 0.447 \\
\hline Poly unsaturated fatty acid & $16.8(7.9)$ & $18.2(7.5)$ & 0.063 \\
\hline Fibre $(\mathrm{g} / \mathrm{d})$ & $36.1(19.0)$ & $60.7(28.2)$ & $<0.001$ \\
\hline Fructose(g/d) & $20.5(8.2)$ & $16.8(9.8)$ & $<0.001$ \\
\hline Caffeine(mg/d) & $145.6(116.4)$ & $126.2(107.0)$ & 0.079 \\
\hline Iron(mg/d) & $49.3(29.5)$ & $25.7(14.0)$ & $<0.001$ \\
\hline Vitamin C(mg/d) & $127.0(80.3)$ & $154.0(70.4)$ & 0.001 \\
\hline Vitamin E(mg/d) & $11.6(5.7)$ & $12.9(4.9)$ & 0.019 \\
\hline Vitamin B12(g/d) & $3.8(3.4)$ & $4.1(2.4)$ & 0.303 \\
\hline Vitamin D(g/d) & $1.5(1.1)$ & $2.0(1.7)$ & $<0.001$ \\
\hline Food groups & & & $<0.001$ \\
\hline Total dairy (g/d) & $315.9(191.6)$ & $424.9(246.0)$ & $<0.001$ \\
\hline Whole grain (g/d) & $102.2(118.4)$ & $163.7(168.2)$ & $<0.001$ \\
\hline Refined grain(g/d) & $428.7(246.5)$ & $328.6(170.3)$ & $<0.001$ \\
\hline Red and processed meat(g/d) & $0.8(0.7)$ & $0.4(0.4)$ & $<0.001$ \\
\hline Fruits(g/d) & $342.1(172.0)$ & $333.9(257.5)$ & 0.687 \\
\hline Vegetables(g/d) & $232.9(109.4)$ & $287.0(170.1)$ & $<0.001$ \\
\hline
\end{tabular}

$P$ values are resulted from student t-test

Table 3 The association between Tertiles of dietary total antioxidant capacity (DTAC) and risk of HP infection among the participants of study. 


\begin{tabular}{|c|c|c|c|c|}
\hline & \multicolumn{3}{|c|}{ Tertiles of dietary total antioxidant capacity } & $P$ for trend \\
\hline & $\mathrm{T} 1$ & $\mathrm{~T} 2$ & T3 & \\
\hline \multicolumn{5}{|l|}{ DTAC } \\
\hline Case/Total & $20 / 120$ & $46 / 147$ & $84 / 185$ & \\
\hline Crude & 1.00 (Ref) & $0.93(0.48-1.77)$ & $0.29(0.14-0.61)$ & 0.001 \\
\hline Model 1* & 1.00 (Ref) & $0.76(0.52-1.12)$ & $0.37(0.24-0.58)$ & $<0.001$ \\
\hline Model 2t & 1.00 (Ref) & $0.74(0.42-1.28)$ & $0.20(0.10-0.40)$ & $<0.001$ \\
\hline
\end{tabular}

${ }^{\star}$ Model 1: adjusted for age, sex.

${ }^{\dagger}$ Model 2: adjusted for model 1 and BMI, waist circumference, physical activity, smoking, dietary intake of energy and fat. 